\title{
8 Das lokalisierte Prostatakarzinom
}

\author{
Sigrun Holze, Norbert Köhler, Lutz Gansera, Jens-Uwe Stolzenburg, \\ Elmar Brähler und Oliver Gralla
}

\begin{abstract}
Das Prostatakarzinom ist der häufigste bösartige Tumor des Mannes. Pro Jahr werden in Deutschland mehr als 60.000 Fälle neu diagnostiziert (RKI 2010). Erkrankungen treten selten vor dem 50. Lebensjahr auf. Aufgrund erweiterter Diagnosemöglichkeiten werden die Tumoren heute zunehmend in einem frühen, lokal begrenzten Stadium entdeckt. In Verbindung mit verbesserten Behandlungsmethoden steigen die Heilungschancen dadurch beträchtlich an.

Im Fokus der Therapiediskussion steht zunehmend auch die postoperative Lebensqualität der Patienten. Dies spielt beim Prostatakarzinom eine umso bedeutendere Rolle, da hier eine Vielzahl an Behandlungsoptionen zur Verfügung steht. Die individuelle Therapiewahl ist daher eine wichtige und nicht weniger schwierige Entscheidung.
\end{abstract}

\subsection{Therapieoptionen beim lokal begrenzten Prostatakarzinom}

Zu den typischen Behandlungsmethoden beim organbegrenzten Prostatakrebs gehören die operativen und strahlentherapeutischen Verfahren. Hinzu kommen hormonelle sowie abwartende Therapieoptionen (Heidenreich et al. 2008; Wirth et al. 2009):

- Operation (retropubisch, perineal, laparoskopisch/endoskopisch, roboterassistiert)

- Strahlentherapie (perkutan, Brachytherapie)

- Hormontherapie

- abwartende Überwachung (Active Surveillance, Watchful Waiting)

\subsubsection{Operation (radikale Prostatektomie)}

Bei der Operation wird die Prostata, die zwischen Harnröhre und Blase liegt, samt Samenblasen und Anteilen der Samenleiter entfernt (s. Abb. 1). Anschließend wird die Blase mit dem Harnröhrenstumpf wieder vernäht. Dieses Prinzip ist bei allen 
operativen Eingriffen gleich. Abhängig vom Befund werden in einigen Fällen die Lymphknoten im kleinen Becken ebenfalls entfernt.

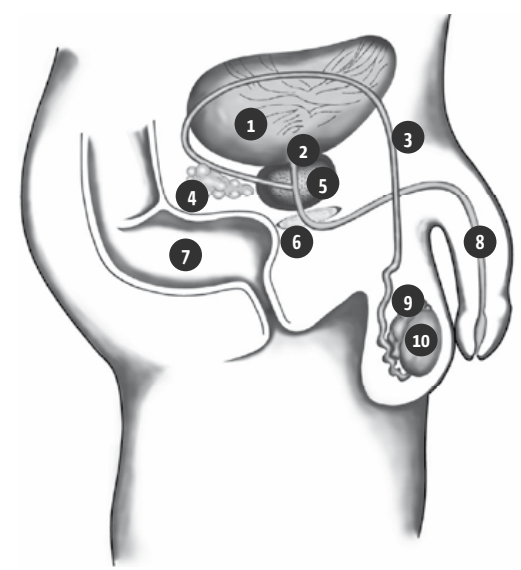

Abb. 1 Anordnung der Organe im kleinen Becken, 1: Blase, 2: Blasenhals, 3: Samenleiter, 4: Samenblase, 5: Prostata, 6: äußerer Schließmuskel, 7: Enddarm, 8: Harnröhre, 9: Nebenhoden, 10: Hoden (mit freundlicher Genehmigung der Universität Münster)

Für die Durchführung der radikalen Prostatektomie (RPE) stehen verschiedene Verfahren zur Verfügung. Sie unterscheiden sich insbesondere durch den Zugangsweg zur Prostata (Kirby et al. 2007).

Die offene retropubische RPE wird über einen Bauchschnitt zwischen Nabel und Schambein durchgeführt.

Bei der perinealen RPE erfolgt der Eingriff hingegen über einen Schnitt am Damm (zwischen Anus und Peniswurzel). Diese Methode wird heute eher selten durchgeführt.

Neben den offenen Verfahren haben sich zunehmend Patienten schonende, minimal invasive (laparoskopische/endoskopische) Verfahren durchgesetzt. Hierbei werden lange, dünne chirurgische Instrumente sowie eine Stabkamera für die gesamte Operation verwendet. Das Kamerabild wird vergrößert auf einem Monitor abgebildet. Bei der LRPE (laparoskopische RPE) werden die Instrumente über kleine Hülsen in die Bauchhöhle direkt eingebracht. Bei der EERPE (endoskopische extraperitoneale RPE) wird ein künstlicher Raum vor der Blase geschaffen, in den dann die Instrumente eingebracht werden (Stolzenburg et al. 2007). Die Bauchhöhle mit den in ihr liegenden Organen wird nicht tangiert. Endoskopische Verfahren können heute auch roboterunterstützt vorgenommen werden. Hierbei lenkt der Operateur die Roboterarme.

Wenn die Ausdehnung und Aggressivität des Tumors es erlauben, kann der Operateur die für die Erektion erforderlichen Nerven unabhängig vom Operationsverfahren erhalten. Eine Wiedererlangung der Erektionsfähigkeit nach der Operation ist dadurch bei vielen Patienten möglich. 


\subsubsection{Strahlentherapie (Radiatio)}

Lokalisierte Prostatakarzinome sind prinzipiell strahlensensibel, sodass auch hier verschiedene Therapieoptionen zur Auswahl stehen (Sauer 2010).

Bei der perkutanen Radiatio wird die Prostata von außen durch die Haut mit energiereichen radioaktiven Teilchen bestrahlt. Mittlerweile kann die Strahlung so zielgerichtet abgegeben werden, dass umliegendes Gewebe nicht oder nur minimal Schaden nimmt. Die Strahlentherapie wird in verschiedenen Sitzungen durchgeführt.

Daneben besteht die Option einer inneren Bestrahlung (Brachytherapie). Hierbei lassen sich zwei Verfahren unterscheiden (Martin et al. 2010).

Bei der HDR-Brachytherapie (High-Dose-Rate) werden bei dem narkotisierten Patienten über den Damm lange Hohlnadeln in die Prostata eingebracht, über die reiskorngroße Strahlenquellen im Zielorgan verteilt werden. Die Strahlung wird dabei innerhalb kurzer Zeit mit sehr hoher Dosisrate abgegeben. Dieses Verfahren wird oft zweimal durchgeführt und mit einer perkutanen Strahlentherapie kombiniert. Es eignet sich eher für aggressivere lokale Tumoren.

Bei der LDR-Brachytherapie (Low-Dose-Rate) wird die Strahlung über einen längeren Zeitraum mit einer niedrigen Dosisrate abgegeben. Die radioaktiv geladenen Metallstifte (Seeds) verbleiben dauerhaft in der Prostata. Das Verfahren eignet sich besonders für kleinere und weniger aggressive Tumoren.

In bestimmten Fällen kann zudem nach einer operativen Entfernung der Prostata eine zusätzliche Bestrahlung empfohlen werden (ebd.). Dies geschieht insbesondere dann, wenn der Tumor die Kapsel der Prostata überschritten hat oder bei der Operation das Tumorgewebe nicht vollständig entfernt werden konnte.

\subsubsection{Hormontherapie}

Das Prostatakarzinom ist in den ersten Jahren hormonabhängig. In bestimmten Situationen kann ein lokalisiertes Karzinom dementsprechend auch hormonell therapiert werden. Allerdings wird dabei der Krebs nicht geheilt. Daher wird diese Methode nur bei Männern empfohlen, die eine geringere Lebenserwartung haben oder an schweren Begleiterkrankungen leiden, sodass andere Therapieverfahren nicht durchgeführt werden können.

\subsubsection{Abwartendes Verhalten (Active Surveillance und Watchful Waiting)}

Operation, Strahlen- und Hormontherapie sind mit möglichen Komplikationen verbunden. Unter bestimmten Voraussetzungen kann es daher sinnvoll sein, zunächst keine aktive Therapie, sondern eine abwartende Strategie zu wählen.

Die aktive Überwachung (Active Surveillance) sollte dann ins Gespräch gebracht werden, wenn der Tumor nicht oder nur sehr langsam wächst und in absehbarer Zeit kein Progress zu erwarten ist (low risk). Der Status des Tumors wird dabei regelmäßig untersucht, sodass bei einem Fortschreiten eine entsprechende operative oder strahlentherapeutische Behandlung erfolgen kann. 
Im Gegensatz dazu wird beim langfristigen Beobachten (Watchful Waiting) nicht der Tumor selbst, sondern das tumorabhängige Beschwerdebild des einzelnen Patienten überwacht. Eine kurative Therapie wird bei dieser Strategie nicht stattfinden. Erst wenn der Tumor Beschwerden verursacht, werden diese gezielt therapiert. Diese Behandlungsoption sollte nur bei Patienten angewendet werden, deren körperliche Verfassung eine heilende Therapie nicht mehr zulässt oder die Risiken eines kurativen Eingriffs den Nutzen übersteigen würden.

\subsection{Auswirkungen auf die Lebensqualität der Patienten}

Die Lebensqualität spielt eine wichtige Rolle in der Bewertung des Therapieerfolges. Sie wird bei Prostatakarzinom-Patienten oft mit behandlungsbezogenen Folgen wie Inkontinenz und Impotenz in Zusammenhang gebracht (Malcolm et al. 2010). Neben diesen prostataspezifischen Problemen sind jedoch auch generelle gesundheitsbezogene Beeinträchtigungen von Bedeutung (Eton et al. 2002).

\subsubsection{Inkontinenz}

Inkontinenz ist eine häufige, jedoch zumeist temporäre Nebenwirkung nach einer aktiven Prostatakrebstherapie. Sie äußert sich dadurch, dass Urin unkontrolliert austritt. Dies geschieht insbesondere unter körperlicher Belastung, beim Husten, Niesen oder Pressen. Man spricht auch von einer Stress- oder Belastungsinkontinenz.

Oft ist es möglich, diese Störung durch konsequentes Beckenbodentraining zu beheben. Wie schnell eine Wiedererlangung der Kontinenz erfolgt, ist von Mann zu Mann unterschiedlich. Bei manchen Betroffenen dauert es wenige Wochen, bei anderen Monate. Nach einem Jahr hat die Mehrheit der Männer keine Probleme mehr. In einigen Fällen - insbesondere bei älteren Männern - kann die Symptomatik jedoch auf niedrigem Niveau persistieren (Verlust von einzelnen Tropfen Urin). Bei stärkerem Urinverlust stehen verschiedene Operationsverfahren (Schlingen-OP bis hin zum künstlichen Schließmuskel) zur Verfügung.

\subsubsection{Impotenz und sexuelle Störungen}

Eine mögliche Nebenwirkung der Therapie sind sexuelle Funktionsstörungen - unabhängig davon, ob eine Operation, eine Strahlentherapie oder eine hormonelle Therapie gewählt wird. Eine befriedigende Sexualität ist eine wichtige Komponente des subjektiven Wohlbefindens. Patienten mit erektiler Dysfunktion (unzureichende Gliedversteifung) fühlen sich häufig weniger männlich und erleben eine massive Beeinträchtigung ihres Selbstbildes (Oliffe 2005). Die Wiedererlangung der Erektionsfähigkeit ist deshalb neben der Kontinenz eine der wesentlichen Herausforderungen nach der Therapie, sofern eine nerverhaltende Operation überhaupt möglich war.

Unmittelbar an der Prostata verlaufen Gefäß- und Nervenstrukturen, die für eine Erektion notwendig sind. Wird die Funktion der Nerven bei der Operation oder durch die Strahlenwirkung gestört, entfällt die Reizweiterleitung zur Eröffnung der Blutgefäße im Schwellkörper. Dementsprechend bleibt der Bluteinstrom in den Schwell- 
körper aus, der Voraussetzung für die Entstehung einer Erektion ist. Im Gegensatz zur Operation, bei der eine Impotenz unmittelbar nach der Behandlung auftritt, entstehen Erektionsprobleme bei der Strahlentherapie oft verzögert. Andererseits kann sich die postoperative Störung wieder regenerieren, wobei die Problematik nach der Strahlentherapie eher nicht reversibel ist. Langfristig ist die Zahl der Patienten, die an einer Erektionsstörung leiden, nach operativen und strahlentherapeutischen Methoden jedoch vergleichbar (Wirth et al. 2009).

Bei den hormonellen Therapien wird das männliche Sexualhormon Testosteron auf Kastrationsniveau herabgesenkt. Neben einem deutlichen Abfall der Libido ist so langfristig auch ein funktioneller Rückgang des Schwellkörpergewebes im Penis zu verzeichnen. Beides ruft Erektionsstörungen hervor.

Auch bei erektilen Störungen nach der Therapie können sexuelle Lust und Orgasmuserleben erhalten bleiben. Sie werden durch einen so einschneidenden Eingriff allerdings häufig beeinflusst. Mit der Entfernung der Prostata kann zudem keine Samenflüssigkeit mehr produziert werden, wodurch es zu einem „trockenen Orgasmus“ kommt.

Hinzu kommt, dass viele Patienten durch die Krebsdiagnose und ihre Folgen psychisch belastet sind. Dies kann sich auf die Sexualität und die Partnerschaft auswirken. Eine offene Thematisierung dieser Problematik mit dem behandelnden Arzt und der Partnerin ist für viele Patienten hilfreich.

Grundsätzlich gilt bei allen Therapiemethoden: Die Ausdehnung und Aggressivität des Tumors, das Alter des Patienten und seine Erektionsfähigkeit vor der Behandlung haben einen wesentlichen Einfluss auf das Risiko für Erektionsstörungen nach der Therapie. Je jünger der Patient ist und je besser seine Erektionsfähigkeit vor dem Eingriff war, desto günstiger ist in der Regel die Chance für einen Potenzerhalt. Eine frühzeitige Diagnose des Tumors begünstigt zudem die Möglichkeit einer Operation mit Nerverhalt.

\subsubsection{Allgemeine gesundheitsbezogene Lebensqualität}

Sexuelle Dysfunktion und Inkontinenz sind viel diskutierte Probleme nach der Prostatakrebsbehandlung. Daneben berichten Patienten jedoch auch in anderen Bereichen von signifikanten Beeinträchtigungen (Harden et al. 2008; Zenger et al. 2009). So leiden die betroffenen Männer verstärkt unter Fatigue und Schlafstörungen. Oft zeigen sich zudem erhöhte emotionale Belastungen. Diese sind vor der Therapie besonders ausgeprägt.

Es ist erforderlich, all diese Probleme frühzeitig mit dem Patienten zu besprechen. Eine adäquate Beratung über Belastungen und Nebenwirkungen der Therapie ist Grundlage eines ganzheitlichen Behandlungskonzeptes. 


\subsection{Therapien zur Wiedererlangung der Erektionsfähigkeit}

Kommt es nach der Therapie zu Erektionsstörungen, gibt es verschiedene Möglichkeiten zu deren Behandlung (s. Kap. II.6). So existieren potenzsteigernde Medikamente, die sog. PDE-5-Hemmer Levitra ${ }^{\circledR}$, Cialis ${ }^{\circledR}$ und Viagra ${ }^{\circledR}$. Diese sind auch bei älteren Patienten unter Berücksichtigung der Kontraindikationen in der hohen Dosierung gut verschreibbar. Kontrovers wird noch diskutiert, inwieweit eine täglich Einnahme dieser Medikamente unmittelbar nach der Therapie hilft, um die Chance zur Wiedererlangung der Potenz zu erhöhen (Montorsi et al. 2008; Zumbé et al. 2008; McMahon 2006).

Hilft die orale Medikation nicht, kann eine intracavernöse Injektion gute Erfolge erzielen. Hierbei werden gefäßerweiternde Medikamente, die eine Erektion auslösen, völlig schmerzfrei vom Patienten selbst in den Schwellkörper appliziert. Bei dieser Methode ist es unerheblich, ob eine Nerverhaltung durchgeführt wurde oder nicht. Der Erfolg beider Methoden, oral und intracavernös, kann durch die Verwendung von flexiblen Penisringen evtl. noch deutlich verbessert werden.

Des Weiteren besteht die Möglichkeit, eine ausreichende Erektion mit Hilfe einer Vakuumpumpe zu erzielen. Hierbei wird ebenfalls ein Penisring zur Verhinderung des Blutabstroms um den Penis gelegt.

Sollte durch die beschriebenen Verfahren keine zufriedenstellende Erektion erzielt werden können, kann die Implantation von artifiziellen Schwellkörpern (sog. Penisprothese) diskutiert werden. Bei den hydraulischen Prothesen werden mit Flüssigkeit füllbare Zylinder in die Schwellkörper eingelegt, die über ein Schlauchsystem mit einem Pumpmechanismus und einem Reservoir verbunden sind. Diese Operation stellt mit Sicherheit die invasivste Methode dar. Studien zur Lebensqualität zeigen jedoch positive Ergebnisse sowohl in der Befragung der Patienten als auch ihrer Partnerinnen (Burns-Cox et al. 1997).

\subsection{Entscheidungshilfen zum geeigneten Therapieverfahren}

Die Entscheidung für eine bestimmte Behandlungsmethode wird von verschiedenen Faktoren beeinflusst. Hierzu gehören die Art und Ausdehnung des Tumors, der körperliche Allgemeinzustand und das Alter des Patienten, das Erfahrungsprofil des behandelnden Arztes sowie die Präferenzen des Patienten. Ob Bestrahlung, offene, endoskopische oder roboter-assistierte Operation: Es gibt für jede Option hervorragende Zentren mit vergleichbar guten Ergebnissen.

Da kurative Therapien meist dann anstehen, wenn die Lebenserwartung mehr als 1o Jahre beträgt, wird die Wahl für eine der Therapieoptionen beträchtliche Folgen für das weitere Leben nach sich ziehen. Die operativen und strahlentherapeutischen Verfahren sind grundsätzlich kurativ, die Hormontherapie und die abwartenden Methoden hingegen nicht. Eine Tumorkontrolle kann mit dem richtigen Verfahren für viele Jahre nahezu garantiert werden.

Aufgrund der Natur des Prostatakarzinoms muss keine überhastete Therapieentscheidung erfolgen. Vielmehr sollten sich betroffene Männer ausreichend Zeit zur Information nehmen und bei Bedarf verschiedene Meinungen einholen. $\mathrm{Zu}$ viele Informationen, gerade im Internet, können jedoch verwirren. Zentral ist es daher, sich 
gemeinsam mit dem „Urologen des Vertrauens“ und dem Lebenspartner ausreichend intensiv über alle Fragen auszutauschen.

Dabei sollten Fragen zur Lebensqualität und die Projektion der geplanten Lebensumstände eine wichtige Rolle spielen: Wird die Sexualität nach der Therapie weiter eine wichtige Rolle spielen? Bin ich ein aktiver Mensch der wandert, reist und viele soziale Kontakte pflegt? Kann ich damit umgehen, infolge von Watchful Waiting oder Active Surveillance einen Tumor Jahre in mir zu tragen, ohne ihn zu behandeln? Welchen Stellenwert erhält die Lebensqualität im Vergleich zur onkologischen Tumorkontrolle?

\section{Literatur}

Burns-Cox N, Burston A, Gingell IC (1997) Fifteen years experience of penile prosthesis insertion. Int I Impot Res 9, 211-216

Eton DT, Lepore SI (2002) Prostate cancer and health-related quality of life: A review of the literature. PsychoOncology 11, 307-326

Harden I, Northouse L, Climprich B, Pohl JM, Liang I, Kershaw T (2008) The influence of developmental life stage on quality of life in survivors of prostate cancer and their partners. I Cancer Surviv 2, 84-94

Heidenreich A, Aus G, Bolla M, Joniau S, Matveev VB, Schmid HP, Zattoni F (2008) EAU guidelines on prostate cancer. Eur Urol 53, 68-80

Kirby R, Montorsi F, Gontero P, Smith JA (2007) Radical prostatectomy: From open to robotic. Informa Healthcare, London

Malcolm JB, Fabrizio MD, Barone BB, Given RW, Lance RS, Lynch DF, Davis JW, Shaves ME, Schellhammer PF (2010) Quality of life after open or robotic prostatectomy, cryoablation or brachytherapy for localized prostate cancer. J Urol 183, 1822-1828

Martin T, Wenz F, Böhmer D, Sedlmayer F, Hinkelbein W, Henkel TO, Miller K, Wiegel T (2010) Strahlentherapie des Prostatakarzinoms in der neuen S3-Leitlinie. Teil 2: Postoperative Strahlentherapie und Brachytherapie. Urologe 49, 216-220

McMahon CG (2006) Treatment of erectile dysfunction with chronic dosing of Tadalafil. Eur Urol 50, 215-217

Montorsi F, Brock G, Lee I, Shapiro JA, Van Poppel H, Graefen M, Stief C (2008) Effect of nightly versus on-demand Vardenafil on recovery of erectile function in men following bilateral nerve-sparing radical prostatectomy. Eur Urol 54, 924-931

Oliffe I (2005) Constructions of masculinity following prostatectomy - induced impotence. Social Science \& Medicine 60, 2249-2259

RKI - Robert-Koch-Institut, Gesellschaft der epidemiologischen Krebsregister in Deutschland e.V. (2010) Krebs in Deutschland 2005/2006. Häufigkeiten und Trends. Robert-Koch-Institut, Berlin

Sauer R (2010) Strahlentherapie in der Onkologie. Elsevier, Urban \& Fischer, München

Stolzenburg J-U, Gettman MT, Liatsikos EN (2007) Endoscopic extraperitoneal radical prostatectomy. Laparoscopic and robot-assisted surgery. Springer, Berlin

Wirth M, Weißbach L, Ackermann R, Alberti W, Albrecht C, Göckel-Beining B, Fröhner M, Hinkelbein W, Miller K, Rübben H, Wiegel T, Wolff J, Wörmann B (2009) Interdisziplinäre Leitlinie der Qualität S3 zur Früherkennung, Diagnose und Therapie der verschiedenen Stadien des Prostatakarzinomes. Deutsche Gesellschaft für Urologie e.V., Düsseldorf

Zenger M, Hinz A, Stolzenburg J-U, Rabenalt R, Schwalenberg T, Schwarz R (2009) Health-related quality of life of prostate cancer patients compared to the general German population: Age-specific results. Urol Int 83 , 166-170

Zumbé J, Porst H, Sommer F, Grohmann W, Beneke M, Ulbrich E (2008) Comparable Efficacy of Once Daily Versus On-Demand Vardenafil in Men with Mild-to-Moderate Erectile Dysfunction: Findings of the RESTORE Study. Eur Urol 54, 204-212 